\title{
ON THE TRIPLE TENSOR PRODUCT OF NILPOTENT LIE ALGEBRAS
}

\author{
AFSANEH SHAMSAKI AND PEYMAN NIROOMAND
}

\begin{abstract}
In this paper, we give the explicit structure of $\otimes^{3} H$ and $\wedge^{3} H$ where $H$ is a generalized Heisenberg Lie algebra of rank at most 2. Moreover, for a non-abelian nilpotent Lie algebra $L$, we obtain an upper bound for the dimension of $\otimes^{3} L$
\end{abstract}

\section{INTRODUCTION}

It is known from [8, Theorem 3.1] that the dimension of tensor product of a Lie algebra $L$ of dimension $n$ when $\operatorname{dim} L^{2}=m$ is bounded by $(n-m)(n-1)+2$ and the structure of the tensor product of a Heisenberg Lie algebra is given. Let $H$ be a generalized Heisenberg Lie algebra of rank at most 2. In this paper, we complete the explicit structure of the tensor square and the exterior square of $H$. Moreover, we give the triple tensor product and the triple exterior product of $H$. Finally, we show that dimension of the triple tensor of a Lie algebra $L$ with derived subalgebra of dimension $m$ is bounded by $n(n-m)^{2}$.

\section{PRELIMINARY AND KNOWN RESUltS}

Let $\mathbb{F}$ be a fixed field and [,] is used to denote the Lie bracket. For any two Lie algebras $L$ and $K$, we say there exists an action $L$ on $K$ if an $\mathbb{F}$-bilinear map $L \times K \rightarrow K,(l, k) \mapsto{ }^{l} k$ satisfying

$$
{ }^{\left[l, l^{\prime}\right]} k={ }^{l}\left({ }^{l^{\prime}} k\right)-{ }^{l^{\prime}}\left({ }^{l} k\right) \quad \text { and } \quad{ }^{l}\left[k, k^{\prime}\right]=\left[{ }^{l} k, k^{\prime}\right]+\left[k,{ }^{l} k^{\prime}\right]
$$

for all $l, l^{\prime} \in L$ and $k, k^{\prime} \in K$.

The actions are compatible if

$$
{ }^{l} l^{\prime}=\left[l^{\prime},{ }^{k} l\right] \quad \text { and } \quad{ }^{k} l k^{\prime}=\left[k^{\prime},{ }^{l} k\right]
$$

for all $k, k^{\prime} \in K, l, l^{\prime} \in L$.

Clearly, a Lie algebra $L$ acts on itself by ${ }^{l^{\prime}} l=\left[l^{\prime}, l\right]$ for all $l, l^{\prime} \in L$. Morover, for all Lie algebras $Q$, a bilinear function $\varphi: L \times K \rightarrow Q$ is called a Lie pairing if for all

2020 Mathematics Subject Classification. 17B30, 17B05, 17B99.

Key words and phrases. Schur multiplier; nilpotent Lie algebra, capable Lie algebra, triple tensor product.

Corresponding Author: Peyman Niroomand. 
$l, l^{\prime} \in L$ and $k, k^{\prime} \in K$, we have

$$
\begin{aligned}
& \varphi\left(\left[l, l^{\prime}\right], k\right)=\varphi\left(l,^{l^{\prime}} k\right)-\varphi\left(l^{\prime},{ }^{l} k\right), \\
& \varphi\left(l,\left[k, k^{\prime}\right]\right)=\varphi\left({ }^{k^{\prime}} l, k\right)-\varphi\left({ }^{k} l, k^{\prime}\right), \\
& \varphi\left({ }^{k} l,^{l^{\prime}} k^{\prime}\right)=-\left[\varphi(l, k), \varphi\left(l^{\prime}, k^{\prime}\right)\right] .
\end{aligned}
$$

The non-abelian tensor product $L \otimes K$ is the Lie algebra generated by symbols $l \otimes k$ for all $l \in L$ and $k \in K$ with the following defining relations

$$
\begin{aligned}
& c(l \otimes k)=c l \otimes k=l \otimes c k, \\
& \left(l+l^{\prime}\right) \otimes k=l \otimes k+l^{\prime} \otimes k, \\
& l \otimes\left(k+k^{\prime}\right)=l \otimes k+l \otimes k^{\prime}, \\
& {\left[l, l^{\prime}\right] \otimes k=l \otimes l^{\prime} k-l^{\prime} \otimes{ }^{l} k,} \\
& l \otimes\left[k, k^{\prime}\right]={ }^{k^{\prime}} l \otimes k-{ }^{k} l \otimes k^{\prime}, \\
& {\left[(l \otimes k),\left(l \otimes k^{\prime}\right)\right]=-{ }^{k} l \otimes{ }^{l^{\prime}} k^{\prime}}
\end{aligned}
$$

for all $c \in \mathbb{F}, l, l^{\prime} \in L$ and $k, k^{\prime} \in K$. If $L=K$ and all actions are Lie multiplication, then $L \otimes L$ is called the non-abelian tensor square of $L$. The next two propositions are used in the rest of paper.

Proposition 2.1. 3, Proposition 3] There are actions of both $L$ and $K$ on $L \otimes K$ given by

$$
\begin{aligned}
& l^{\prime}(l \otimes k)=\left[l, l^{\prime}\right] \otimes k+l \otimes\left({ }^{\prime} k\right), \\
& k^{\prime}(l \otimes k)=\left({ }^{k^{\prime}} l\right) \otimes k+l \otimes\left[k^{\prime}, k\right]
\end{aligned}
$$

for all $l, l^{\prime} \in L$ and $k, k^{\prime} \in K$.

Recall that a Lie algebra $H$ is called a generalized Heisenberg of rank $n$ if $H^{2}=$ $Z(H)$ and $\operatorname{dim} H^{2}=n$. If $n=1$, then $H$ is a Heisenberg Lie algebra that is more well-known. Such Lie algebras are odd dimension and $H \cong H(m)=\left\langle x_{i}, y_{i}, z\right|$ $\left.\left[x_{i}, y_{i}\right]=z, 1 \leq i \leq m\right\rangle$.

Proposition 2.2. [8, Proposition 3.3] Let $H(m)$ be a Heisenberg Lie algebra. Then

$$
H(m) \otimes H(m) \cong H(m) / H(m)^{2} \otimes H(m) / H(m)^{2}
$$

for all $m$ such that $m \geq 2$. In the case $m=1, H(1) \otimes H(1)$ is an abelian Lie algebra of dimension 6.

Let $L \square L$ be the submodule of $L \otimes L$ generated by the elements $l \otimes l$. Then the exterior square $L \wedge L$ of $L$ is the quotient $L \wedge L \cong L \otimes L / L \square L$. For all $l \otimes l^{\prime} \in L \otimes L$, we denote the coset $l \otimes l^{\prime}+L \square L$ by $l \wedge l^{\prime}$.

The Schur multiplier of a Lie algebra $L$ is defined as $\mathcal{M}(L) \cong R \cap F^{2} /[R, F]$ where $L \cong F / R$ and $F$ is a free Lie algebra. It is known that the Lie algebra $\mathcal{M}(L)$ is abelian and independent of the choice of the free Lie algebra $F$ (see 1, 2] for more information).

Lemma 2.3. [5, Lemma 23] Let $L$ be an $n$-dimensional abelian Lie algebra. Then $\operatorname{dim} \mathcal{M}(L)=\frac{1}{2} n(n-1)$.

Lemma 2.4. [6, Lemma 2.8] Let $L$ be a nilpotent Lie algebra of class two. Then $L \wedge L \cong \mathcal{M}(L) \oplus L^{2}$. 
Let $A(n)$ be used to denote an abelian Lie algebra of dimension $n$. Then we have

Lemma 2.5. 11, Corollary 2.5 and Lemma 3.2]

(i). $A(n) \wedge A(n) \cong \mathcal{M}(A(n))$,

(ii). $H(1) \wedge H(1) \cong A(3)$,

(iii). $H(m) \wedge H(m) \cong A\left(2 m^{2}-m\right)$ for all $m \geq 2$.

The next theorem shows that the exterior product of a Lie algebra $L$ is a direct summand of $L \otimes L$ when $L / L^{2}$ is of finite dimension.

Theorem 2.6. 9, Theorem 2.5] Let $L / L^{2}$ be a finite dimensional Lie algebra. Then

$$
L \otimes L \cong L \wedge L \oplus L \square L .
$$

In the following, the notations $L_{6,22}(\varepsilon), L_{5,8}, L_{6,7}^{(2)}(\eta)$ and $L_{1}$ are taken from [4] and 10 .

Proposition 2.7. 6. Theorem 2.15] The Schur multiplier of Lie algebras $L_{6,22}(\varepsilon)$, $L_{5,8}, L_{6,7}^{(2)}(\eta)$, and $L_{1}$ are abelian Lie algebras of dimension 8, 6, 8 and 9, respectively.

A Lie algebra $L$ is called capable provided that $L \cong H / Z(H)$ for a Lie algebra $H$.

Proposition 2.8. [10, Proposition 3.1] Let $H$ be a non-capable generalized Heisenberg Lie algebra of rank 2 such that $\operatorname{dim} H=n$. Then

$$
\operatorname{dim} \mathcal{M}(H)=\frac{1}{2}(n-3)(n-2)-2
$$

or

$$
\operatorname{dim} \mathcal{M}(H)=\frac{1}{2}(n-1)(n-4)+1
$$

\section{MAIN RESULTS}

We know from Proposition 2.1 $L$ acts on $L \otimes L$. On the other hand, the tensor product $L \otimes L$ acts on $L$ by ${ }^{t} l={ }^{\lambda(t)} l$ for all $t \in L \otimes L$ and $l \in L$ such that $\lambda: L \otimes L \rightarrow L$ is a homomorphism given by $a \otimes b \mapsto[a, b]$. These actions are compatible and we can construct the triple tensor product $\otimes^{3} L=(L \otimes L) \otimes L$. In this section, we give the explicit structure of $L \otimes L=\otimes^{2} L$ and $L \wedge L=\wedge^{2} L$ when $L$ is a generalized Heisenberg of rank 2. Moreover, we obtain $\otimes^{3} L$ and $\wedge^{3} L$ when $L$ is a generalized Heisenberg of rank at most 2. Also, for a non-abelian nilpotent Lie algebra $L$, we give an upper bound for the triple tensor product of $L$.

The following lemmas are useful instruments in the next.

Lemma 3.1. Let $L$ be a Lie algebra of nilpotency class two. Then

(i). $L \otimes L$ acts trivially on $L$.

(ii). $(L \otimes L) \otimes L$ is an abelian Lie algebra.

Proof. (i). We know that $L$ is a Lie algebra of nilpotency class two, so $\gamma_{3}(L)=0$. By considering action of $L \otimes L$ on $L$, we have ${ }^{\lambda(a \otimes b)} c=[[a, b], c]=0$ for all $a \otimes b \in L \otimes L$ and $c \in L$. Therefore $L \otimes L$ acts trivially on $L$. 
(ii). By using the relations of the non-abelian tensor on $(L \otimes L) \otimes L$ and the part (i), we have

$$
\begin{aligned}
{\left[(a \otimes b) \otimes c,\left(a^{\prime} \otimes b^{\prime}\right) \otimes c^{\prime}\right] } & =-\left({ }^{c}(a \otimes b)\right) \otimes a^{a^{\prime} \otimes b^{\prime}} c \\
& =-{ }^{c}(a \otimes b) \otimes 0=0
\end{aligned}
$$

Therefore $\left[(a \otimes b) \otimes c,\left(a^{\prime} \otimes b^{\prime}\right) \otimes c^{\prime}\right]=0$ for all $(a \otimes b) \otimes c,\left(a^{\prime} \otimes b^{\prime}\right) \otimes c^{\prime} \in(L \otimes L) \otimes L$, and so $(L \otimes L) \otimes L$ is an abelian Lie algebra.

Let $L$ be a nilpotent Lie algebra of class $k, i_{L}: L \rightarrow L$ be the identity homomorphism and $\gamma_{k}(L)$ be the $k$-th term of the lower central series of $L$ and $\varphi: \gamma_{k}(L) \rightarrow L$ be a natural homomorphism. Define homomorphisms $\bar{\varphi}=\left(\varphi \otimes i_{L}\right) \otimes i_{L}:\left(\gamma_{k}(L) \otimes\right.$ $L) \otimes L \rightarrow \otimes^{3} L$ and $\gamma:(L \otimes L) \otimes \gamma_{k}(L) \rightarrow \otimes^{3} L$ given by $(a \otimes b) \otimes c \mapsto(a \otimes b) \otimes c$. Then

Lemma 3.2. Let $L$ be a non-abelian nilpotent Lie algebra of class $k$, then $\operatorname{Im} \gamma \subseteq$ $\operatorname{Im} \bar{\varphi}$.

Proof. By using the relation $k \otimes\left[l, l^{\prime}\right]=\left[l^{\prime}, k\right] \otimes l-[l, k] \otimes l^{\prime}$ on $L \otimes L$, we can see that $(a \otimes b) \otimes\left[x_{1}, \ldots, x_{k-1}, x_{k}\right] \in\left(\gamma_{k}(L) \otimes L\right) \otimes L$ for all $a, b, x_{1}, \ldots, x_{k-1}, x_{k} \in L$. Therefore the result follows.

The next result plays a key role in proving the next theorem.

Proposition 3.3. If $L$ is a nilpotent Lie algebra of class $k$, then

$$
\left(\gamma_{k}(L) \otimes L\right) \otimes L \stackrel{\left(\varphi \otimes i_{L}\right) \otimes i_{L}}{\longrightarrow} \otimes^{3} L \longrightarrow \otimes^{3} L / \gamma_{k}(L) \rightarrow 0,
$$

is exact.

Proof. By using Lemma 3.2 and [13, Proposition 1.3], the result follows.

In the following proposition, we are going to determine the structure of $\otimes^{3} H(m)$ and $\wedge^{3} H(m)$ for all $m \geq 1$.

Proposition 3.4. Let $H(m)$ be a Heisenberg Lie algebra. Then

$$
\otimes^{3} H(m) \cong \begin{cases}A(12) & \text { if } m=1 \\ A\left(2^{3} m^{3}\right) & \text { if } m \geq 2\end{cases}
$$

and

$$
\wedge^{3} H(m) \cong \begin{cases}A(2) & \text { if } m=1 \\ A\left(4 m^{3}-4 m^{2}-m\right) & \text { if } m \geq 2\end{cases}
$$

Proof. We claim that $\left(\varphi\left(L^{2}\right) \otimes L\right) \otimes L \cong\left(\varphi\left(L^{2}\right) \otimes L^{a b}\right) \otimes L^{a b}$. It is clear that $\varphi\left(L^{2}\right)$ and $L$ act trivially on each other. Hence $\left(\varphi\left(L^{2}\right) \otimes L\right) \otimes L \cong\left(\varphi\left(L^{2}\right) \otimes L^{a b}\right) \otimes L$. Also, $\varphi\left(L^{2}\right) \otimes L^{a b}$ and $L$ act trivially on each other. Thus $\left(\varphi\left(L^{2}\right) \otimes L\right) \otimes L \cong$ $\left(\varphi\left(L^{2}\right) \otimes L^{a b}\right) \otimes L^{a b}$. By using the following exact sequence

$$
L^{2} \otimes L \stackrel{\varphi \otimes i_{L}}{\longrightarrow} L \otimes L \longrightarrow L / L^{2} \otimes L / L^{2} \longrightarrow 0,
$$

we have

(3.1) $\operatorname{dim} L \otimes L=\operatorname{dim} L^{a b} \otimes L^{a b}+\operatorname{dim} \operatorname{Im} \varphi \otimes i_{L}=\operatorname{dim} L^{a b} \otimes L^{a b}+\operatorname{dim} \varphi\left(L^{2}\right) \otimes L$. 
Let $L \cong H(1)$. Since $H(1) \otimes H(1) \cong A(6)$ and $H(1)^{a b} \otimes H(1)^{a b} \cong A(4)$, we have $\operatorname{dim} \varphi\left(L^{2}\right) \otimes L=2$ by using (3.1). Since $\left(\varphi\left(L^{2}\right) \otimes L\right) \otimes L \cong\left(\varphi\left(L^{2}\right) \otimes L^{a b}\right) \otimes L^{a b}$,

$$
\operatorname{dim}\left(\varphi\left(L^{2}\right) \otimes L\right) \otimes L=\left(\operatorname{dim} \varphi\left(L^{2}\right) \otimes L^{a b}\right) \operatorname{dim} L^{a b} .
$$

Now, by using (3.2), Proposition 3.3 and Lemma 3.1 (ii), we have

$$
\operatorname{dim} \otimes^{3} L=\operatorname{dim} \otimes^{3} L^{a b}+\operatorname{dim}\left(\varphi\left(L^{2}\right) \otimes L^{a b}\right) \otimes L^{a b}=12
$$

and so $\otimes^{3} L \cong A(12)$. Similarly, if $L \cong H(m)$ for all $m \geq 2$, then $\otimes^{3} L \cong A\left(2^{3} m^{3}\right)$. Let $L \cong H(1)$, then $\operatorname{dim} \varphi\left(L^{2}\right) \wedge L=\operatorname{dim} \wedge^{2} L-\operatorname{dim} \wedge^{2} L^{a b}=2$ by using the following exact sequence

$$
L^{2} \wedge L \stackrel{\varphi \wedge i_{L}}{\longrightarrow} L \wedge L \longrightarrow L / L^{2} \wedge L / L^{2} \longrightarrow 0
$$

and Lemma 2.5. We can easily see $\left(\varphi\left(L^{2}\right) \wedge L\right) \wedge L \cong\left(\varphi\left(L^{2}\right) \wedge L^{a b}\right) \wedge L^{a b}$ and since $\varphi\left(L^{2}\right) \wedge L \cong A(2)$, we have $\left(\varphi\left(L^{2}\right) \wedge L\right) \wedge L \cong A(2) \wedge A(2)$. Thus $\left(\varphi\left(L^{2}\right) \wedge L\right) \wedge L \cong A(1)$ by using Lemma 2.5. On the other hand, $\wedge^{3} L / L^{2} \cong(A(2) \wedge A(2)) \wedge A(2)$, hence $\wedge^{3} L / L^{2} \cong A(1)$ by using Lemma 2.5 and [7, Lemma 2.1.7]. Consider the following exact sequence

$$
\left(L^{2} \wedge L\right) \wedge L \stackrel{\left(\varphi \wedge i_{L}\right) \wedge i_{L}}{\longrightarrow} \wedge^{3} L \longrightarrow \wedge^{3} L / L^{2} \rightarrow 0
$$

Therefore

$$
\begin{aligned}
\operatorname{dim} \wedge^{3} L & =\operatorname{dim} \wedge^{3} L / L^{2}+\operatorname{dim} \operatorname{Im}\left(\varphi \wedge i_{L}\right) \\
& =\operatorname{dim} \operatorname{dim} \wedge^{3} L / L^{2}+\operatorname{dim}\left(\varphi\left(L^{2}\right) \wedge L\right) \wedge L \\
& =2 .
\end{aligned}
$$

Since $\otimes^{3} L$ is an abelian Lie algebra by using Lemma 3.1 (ii), we have $\wedge^{3} L \cong A(2)$. Similarly, if $L \cong H(m)$ for all $m \geq 2$, then $\wedge^{3} L \cong A\left(4 m^{3}-4 m^{2}-m\right)$.

The following theorem determines all $n$-dimensional capable generalized Heisenberg Lie algebras of rank 2 .

Theorem 3.5. 10, Theorem 3.6] Let $H$ be a generalized Heisenberg Lie algebra such that $\operatorname{dim} H=n$ and $\operatorname{dim} H^{2}=2$. Then $H$ is capable if and only if $n=5,6$ or 7 and $H \cong L_{5,8}=\left\langle x_{1}, \ldots, x_{5} \mid\left[x_{1}, x_{2}\right]=x_{4},\left[x_{1}, x_{3}\right]=x_{5}\right\rangle, H \cong L_{6,22}(\varepsilon)=$ $\left\langle x_{1}, \ldots, x_{6} \mid\left[x_{1}, x_{2}\right]=x_{5}=\left[x_{3}, x_{4}\right],\left[x_{1}, x_{3}\right]=x_{6},\left[x_{2}, x_{4}\right]=\epsilon x_{6}\right\rangle$ where $\varepsilon \in \mathbb{F} /(\stackrel{*}{\sim})$, $L_{6,7}^{(2)}(\eta)=\left\langle x_{1}, \ldots, x_{6}\right|\left[x_{1}, x_{2}\right]=x_{5},\left[x_{1}, x_{3}\right]=x_{6},\left[x_{2}, x_{4}\right]=\eta x_{6},\left[x_{3}, x_{4}\right]=x_{5}+x_{6}$ where $\eta \in\{0, \omega\}$ or $H \cong L_{1}=\left\langle x_{1}, \ldots, x_{7}\right|\left[x_{1}, x_{2}\right]=x_{6}=\left[x_{3}, x_{4}\right],\left[x_{1}, x_{5}\right]=x_{7}=$ $\left.\left[x_{2}, x_{3}\right]\right\rangle$.

The next propositions determine the structure of $\otimes^{2} H, \wedge^{2} H, \otimes^{3} H$ and $\wedge^{3} H$ when $H$ is a generalized Heisenberg Lie algebra of rank 2 .

Proposition 3.6. Let $H$ be an n-dimensional generalized Heisenberg Lie algebra of rank 2.

(i). If $H$ is non-capable, then

$$
\otimes^{2} H \cong \begin{cases}A\left((n-2)^{2}\right) & \text { if } Z^{\wedge}(H)=H^{2}, \\ A\left((n-2)^{2}+2\right) & \text { otherwise }\end{cases}
$$


and

$$
\wedge^{2} H \cong \begin{cases}A\left(\frac{1}{2}(n-3)(n-2)\right) & \text { if } Z^{\wedge}(H)=H^{2} \\ A\left(\frac{1}{2}(n-1)(n-4)+3\right) & \text { otherwise. }\end{cases}
$$

(ii). If $H$ is capable, then

$$
\otimes^{2} H \cong \begin{cases}A(14) & \text { if } H \cong L_{5,8} \\ A(20) & \text { if } H \cong L_{6,22}(\varepsilon) \text { or } L_{6,7}^{(2)}(\eta) \\ A(26) & \text { if } H \cong L_{1}\end{cases}
$$

and

$$
\wedge^{2} H \cong \begin{cases}A(8) & \text { if } H \cong L_{5,8} \\ A(10) & \text { if } H \cong L_{6,22}(\varepsilon) \text { or } L_{6,7}^{(2)}(\eta) \\ A(11) & \text { if } H \cong L_{1}\end{cases}
$$

Proof. (i). First, we obtain the exterior product of $H$. By invoking Lemma 2.4 and Proposition 2.8, we have $\wedge^{2} H=\mathcal{M}(H) \oplus H^{2} \cong A\left(\frac{1}{2}(n-3)(n-2)\right)$ when $Z^{\wedge}(H)=H^{2}$. By a similar way, we can see that $\wedge^{2} H=A\left(\frac{1}{2}(n-1)(n-4)+3\right)$ when $Z^{\wedge}(H) \neq H^{2}$.

Since $H \square H \cong H^{a b} \square H^{a b} \cong A\left(\frac{1}{2}(n-m)(n-m+1)\right)$ by [9, Lemma 2.3] and using Theorem 2.6. we have $\otimes^{2} H=H \square H \oplus H \wedge H \cong A\left(n^{2}-2 n+3\right)$ when $Z^{\wedge}(H)=H^{2}$. By a similar way, we can see that $\otimes^{2} H \cong A\left(n^{2}-2 n+5\right)$ when $Z^{\wedge}(H) \neq H^{2}$. (ii). The proof is similar to the part (i).

Proposition 3.7. Let $H$ be $n$-dimensional generalized Heisenberg Lie algebra of rank 2.

(i). Let $H$ is non-capable. Then

$$
\otimes^{3} H \cong \begin{cases}A\left((n-2)^{3}\right) & \text { if } Z^{\wedge}(H)=H^{2} \\ A\left(\left(n^{2}-4 n+6\right)(n-2)\right) & \text { otherwise. }\end{cases}
$$

and

$$
\wedge^{3} H \cong \begin{cases}A\left(\frac{1}{2}(n-2)\left(n^{2}-6 n+7\right)\right) & \text { if } Z^{\wedge}(H)=H^{2}, \\ A\left(\frac{1}{2} n\left(n^{2}-8 n+23\right)-14\right) & \text { otherwise. }\end{cases}
$$

(ii). If $H$ is capable, then

$$
\otimes^{3} H \cong \begin{cases}A(42) & \text { if } H \cong L_{5,8} \\ A(80) & \text { if } H \cong L_{6,22}(\varepsilon) \text { or } L_{6,7}^{(2)}(\eta), \\ A(130) & \text { if } H \cong L_{1}\end{cases}
$$

and

$$
\wedge^{3} H \cong \begin{cases}A(12) & \text { if } H \cong L_{5,8} \\ A(20) & \text { if } H \cong L_{6,22}(\varepsilon) \text { or } L_{6,7}^{(2)}(\eta) \\ A(39) & \text { if } H \cong L_{1}\end{cases}
$$

Proof. The proof is obtained by a similar way used in the proof of Proposition 3.4 .

Theorem 3.8. Let $L$ be an n-dimensional non-abelian nilpotent Lie algebra with derived subalgebra of dimension $m$. Then

$$
\operatorname{dim} \otimes^{3} L \leq n(n-m)^{2} .
$$


In particular, for $m=1$ the equality holds if and only if $L \cong H(1)$.

Proof. Let $L$ be an $n$-dimesional nilpotent Lie algebra of nilpotency class $k$. We proceed by induction on $n$. Since $L$ is non-abelian, $n \geq 3$. If $n=3$, then $L \cong H(1)$ by the classification of all nilpotent Lie algebras is given in 4. By using Proposition 3.4. we have $\otimes^{3} H(1) \cong A(12)$. Therefore the result holds. Since $\gamma_{k}(L)$ is central, $\gamma_{k}(L)$ and $L$ act trivially on each other and so we have $\gamma_{k}(L) \otimes L \cong \gamma_{k}(L) \otimes L^{a b}$ by using [3, Proposition 5]. By the same reason and using [3, Proposition 5], we have $\left(\gamma_{k}(L) \otimes L\right) \otimes L \cong\left(\gamma_{k}(L) \otimes L^{a b}\right) \otimes L^{a b}$. Consider two cases.

Let $L / \gamma_{k}(L)$ is abelian. Then $L$ is a nilpotant Lie algebra of class two and $\gamma_{k}(L)=$ $L^{2}$. Thus $\operatorname{dim} \otimes^{3} L / \gamma_{k}(L)=\operatorname{dim} \otimes^{3} L / L^{2}=(n-m)^{3}$. By using Proposition 3.3. we have

$$
\begin{aligned}
\operatorname{dim} \otimes^{3} L & \leq \operatorname{dim} \otimes^{3}\left(L / \gamma_{k}(L)\right)+\operatorname{dim}\left(\left(\gamma_{k}(L) \otimes L\right) \otimes L\right) \\
& =(n-m)^{3}+m(n-m)^{2}=n(n-m)^{2} .
\end{aligned}
$$

If $L / \gamma_{k}(L)$ is non-abelian, then $\operatorname{dim} L / \gamma_{k}(L)=n-\operatorname{dim} \gamma_{k}(L)$ and $\operatorname{dim}\left(L / \gamma_{k}(L)\right)^{2}=$ $m-\operatorname{dim} \gamma_{k}(L)$, hence

$$
\operatorname{dim} \otimes^{3}\left(L / \gamma_{k}(L)\right) \leq\left(n-\operatorname{dim} \gamma_{k}(L)\right)(n-m)^{2}
$$

by using the induction hypothesis. Now, Proposition 3.3 and (3.3) imply that

$$
\begin{aligned}
\operatorname{dim} \otimes^{3} L & \leq \operatorname{dim} \otimes^{3}\left(L / \gamma_{k}(L)\right)+\operatorname{dim}\left(\left(\gamma_{k}(L) \otimes L\right) \otimes L\right) \\
& \leq\left(n-\operatorname{dim} \gamma_{k}(L)\right)(n-m)^{2}+\operatorname{dim} \gamma_{k}(L)(n-m)^{2} \\
& =n(n-m)^{2} .
\end{aligned}
$$

Let $m=1$. Then $L \cong H(k) \oplus A(n-2 k-1)$ for all $k \geq 1$ by using [12, Lemma 3.3]. By using Proposition 3.4 the equality holds if and only if $L \cong H(1)$.

\section{REFERENCES}

[1] Batten, P., Moneyhun, K. and Stitzinger, E.; On characterizing nilpotent Lie algebras by their multipliers. Comm. Algebra 24 (1996), no. 14, 4319-4330.

[2] Batten, P. and Stitzinger, E.; On covers of Lie algebras. Comm. Algebra 24 (1996), no. 14, 4301-4317.

[3] Ellis, G.; A non-abelian tensor product of Lie algebras. Glasgow Mathematical Journal 33, no. 1 (1991), 101-120.

[4] De Graaf, W. A.; Classification of 6-dimensional nilpotent Lie algebras over fields of characteristic not 2. J. Algebra 309, no. 2, (2007), 640-653.

[5] Moneyhun, K.; Isoclinisms in Lie algebras. Algebras Groups Geom. 11 , no. 1, (1994), 9-22.

[6] Johari, F., and Niroomand, P.; Certain functors of nilpotent Lie algebras with the derived subalgebra of dimension two. J. Algebra Appl. 19, no. 01, (2020), 2050012.

[7] Johari, F., Parvizi, M., and Niroomand, P. Capability and Schur multiplier of a pair of Lie algebras. J. Geom. Phys. 114, (2017), 184-196.

[8] Niroomand, P.; On the tensor square of non-abelian nilpotent finite-dimensional Lie algebras. Linear and Multilinear Algebra 59, no. 8 (2011), 831-836.

[9] Niroomand, P.; Some properties on the tensor square of Lie algebras. J. Algebra Appl. 11 (2012), no. 5, 1250085, 6 pp.

[10] P. Niroomand, F. Johari and M. Parvizi, Capable Lie algebras with the derived subalgebra of dimension 2 over an arbitrary field. Linear and Multilinear Algebra 67 , no. 3, (2019), 542-554.

[11] Niroomand, P., Parvizi, M. and Russo, F. G.; Some criteria for detecting capable Lie algebras. J. Algebra 384 (2013), 36-44.

[12] Niroomand, P.; On dimension of the Schur multiplier of nilpotent Lie algebras. Cent. Eur. J. Math. 9 (2011), no. 1, 57-64. 
[13] Salemkar, A.R., Tavallaee, H., Mohammadzadeh, H. and Edalatzadeh, B.; On the non-abelian tensor product of Lie algebras. Linear and Multilinear Algebra 58, no. 3, (2010), 333-341.

School of Mathematics and Computer Science, Damghan University, Damghan, Iran Email address: Shamsaki.afsaneh@yahoo.com

Email address: niroomand@du.ac.ir, p_niroomand@yahoo.com

School of Mathematics and Computer Science, Damghan University, Damghan, Iran 